\title{
The Use of Uavs Groups for the Monitoring of Terrestrial Infrastructure Objects
}

\author{
German G. Sebryakov ${ }^{1, *}$ and Viliam $V$. Insarov ${ }^{1}$ \\ ${ }^{1}$ FGUP “GosNIIAS”, Moscow, Russia
}

\begin{abstract}
The paper discusses the basic principles of UAF application to the task of monitoring the terrestrial areas. The emphasis is made on the problem of controlling the UAVs groups and the distribution of functions between the UAVs in the group and the on-land control centres.
\end{abstract}

\section{Introduction}

Recent developments in the field of intelligent systems made it possible to enlarge UAVs application area. Among those developments are: improved capacity for autonomous flight and flight in groups; reduction of high-precision sensors in size and power consumption, which made it possible to use them on board; improved means of communication between UAVs and between the UAV and base station; etc.

Of particular significance in this context is the application of drones for performing complex tasks, such as traffic surveillance; condition monitoring of oil and gas pipelines or power lines, detection and monitoring of ice jams; data collection on chemical or radioactive contamination of air and terrain surface; engineer reconnaissance missions during or after floods, earthquakes or other natural disasters; as a source of visual information during peacekeeping operations, or evacuation operations; forest fires detection, as information support for firefighting teams, etc.

Special equipment installed on board of the UAV, enables it to conduct operations in the areas of terrain, otherwise inaccessible for humans. For example, highly sensitive radars are able to register movement under piles of debris or avalanches; sensors that register the presence of hazardous substances in the air allow to rapidly define the boundaries of contaminated zone; infrared cameras allow to use the optical recognition of objects in the dark.

It is obvious that for most applications listed above maximum efficiency can be achieved through the use of groups of UAVs as opposed to the use of a single aircraft[1-4].

One more application area of UAV groups should be mentioned here apart from the ones mentioned above is the implementation of so-called task-oriented control architecture. In this case operators interact with a general mission and payload manager, instead of having individually tasking each vehicle. The general manager coordinates the set of tasks for a group of UAVs which typically communicate with one another in a decentralized mode. In a system like that every vechicle in the group can perform it's own task thus improving group's overal situation efficiency and information processing rates.

Group of UAVs, when organized in a form of a spatially distributed system can solve the problem of passive detection of radio-emitting and, above all, moving targets with rather high accuracy [5].

It is believed that a group of drones can be effectively used in the electronic warfare in order to suppress communication channels of the opposing side.

Of course, the methods of organizing the group applications of UAVs in each one of the considered situations have their own particularities. Nevertheless, it is of interest to us to consider some of the general points, common to all the implementations of this approach, mainly because the the most part of tasks described above, have the similar sets of elementary actions to be performed in order to accomplish the task. So, the decomposition into similar subtasks is possible.

\section{Formation of a group of UAVs}

In order to perform tasks that involve cooperative UAV actions following problems should be solved :

1. Organization of the group of agents into a network-like system with UAVs of the same type or subgroups of UAVs of the same type or subgroups containing different types of UAVs and manned aircrafts.

2. Determining the optimal number of agents in the group as a solution of the optimization problem. It is to be assumed that the time intervals between flights and flight trajectories constraints are known and the flow of applications may be considered as a random process.

3. Organization of UAVs group takeoff, gathering agents into cruise formation, maintaining the relative position of the agents within the formation during the cruise flight, return to the base, landing of the agents, etc.

\footnotetext{
Corresponding author: sebr@gosniias.ru
} 
4. Route planning and scheduling with account to it's physical feasibility and global requirements applied to the performance; initial decomposition of tasks into a set of logically related subtasks and further distribution of subtasks between the agents of the group.

5. Creating a software platform for the implementation of mission-planning and task payload management capable of coping with predefined set of subtasks, equiped with a set of protocols for ingroup communication and means to add new functions if needed.

6. Establishing effective communication between group agents and mission control center.

7. Making use of modern multichannel and multispectral on board computer vision systems.

The problems listed above do not include the problems of UAV type selection or selection of it's power unit, on board equipment complex, and so on. The report deliberately focuses on the problems inherent in the use of the group of UAVs in dealing with land surface monitoring tasks.

\section{Some notes}

Let us discuss some of the subjects mentioned in previous sections of this report, and let us take a cooperative UAV surveillance task as an example. The observed object is considered to be immobile and all it's characteristics are known. If the software platform is implemented according to modular approach [1-3], then all the characteristics of the object and the search area should be provided to it as inputs in order to formulate the search task. This task is then loaded to planning modules of all the group agents. As a result those modules produce the initial mission plan and a list of individual flight plans for all the group members, the plan and the list are then dispatched to the network configuration manager.

During the mission some events may happen, such as the introduction of a new UAV into the group, technical failures, changes in the mission objectives e.t.c. which are not foreseen in the initial plan. Those events should be taken into account by the system; it should adapt and rebuild flight plans accordingly. For the numerical evaluation of the effectiveness of subtasks distribution, we introduce the concept of functional criteria. A particular set of criteria is determined by the nature of the task and can be further adjusted without affecting the general applicability of the planning approach.

For example, let's consider two criteria that allow UAVs at the same time not to gather too densely in one area and not to exceed limits of wireless communication.

Lпі - criterion that characterizes the coverage of the given area by surveillance group agents, it depends on the evaluation of the observability of the given area by the i-th UAV in the group.

Lci - criterion that characterizes the quality of connection between the i-th UAV and other UAVs in group.
In this case, the total efficiency of the system at any step is determined by the sum of efficiencies of all $\mathrm{N}$ UAVs in the group, according to the formula

$$
L_{\mathrm{g}}=k_{\mathrm{p}} \sum L_{\mathrm{p} i}+k_{\mathrm{c}} \sum L_{\mathrm{c} i}
$$

where $i=1,2 \ldots, N ; N-$ number of UAVs in the group; $L_{g}-$ group performance criterion; $k_{p}, k_{c}-$ weight coefficients $\left(k_{p}+k_{c}=1\right)$.

The higher the total index $L_{\mathrm{g}}$, the better the quality of the solution according to this criteria. Since control tasks handling is decentralized, it possible that the same subtask will be appointed to several drones. In order to avoid such situations a protocol for the resolution of conflicts should be presented.

Naturally, the appropriate data link digital technologies should be used toprovide the necessary information support.

The use of multi-channel systems for solving problems of detection and recognition of objects and complex scenes has significant advantages over singlechannel versions. It provides:

- better reliability of the information about geometrical parameteres and brightness levels of observed objects;

- detection of camouflaged objects, as it is much more difficult to make an object invisible in different spectral ranges;

- noise immunity, because it is difficult to maintain jamming simultaneously in different spectral ranges;

- performance in presence of shielding objects or the noisy backgrounds;

- recognition of objects in poor weather conditions because sensors with different spectral ranges have different sensitivity in same weather conditions.

\section{The prospects}

The prolems to be solved in the nearest future are the following:

1. Determining the methods of forming a group of UAVs as a integral and stable system with distributed structure.

2. The choice of the optimal degree of UAV autonomy in the group. The degree of operator participation is to be determined by alternative logics such as "operator in the control loop" or "operator above the control loop with the right to make the final decision".

3.The development of a high-performance software for the UAV group management and self-organization tasks.

4. Analysis of the information and find the right job in the structure, providing a virtual exchange of information via wireless communication lines in order to maintain the structure and formation a team and replenishing its lost participants and etc.

5. Development of standards regulating the interaction of the operator with a single unmanned platform or with the group of UAVs at all degrees of interoperability. 
6. Development of technique for training the highly skilled operators capable to provide management, navigation and targeting UAV in a group.

7. The development of on-board systems of highprecision detection, based on the multi-channel opticalelectronic and radar systems for small-size UAVs.

8. Development of an autonomous system of evading potential threats and ensuring the safety of the UAV in flight in relation to the other UAVs and piloted aircrafts, and to the ground obstacles, natural or artifitial .

10. Development of models for simulation the functioning single UAV and UAVs groups.

\section{References}

1. P.O. Skobelev, Mehatronika, avtomatizatciia, upravlenie, 12, 33 (2011)
2. Yu.I. Buryak, V.V. Insarov, Journal of Computer and Systems Sciences International, 45(2), 245-262 (2006)

3. N.V. Kim, I.G. Kry'lov, Trudy` MAI, 62 (2012) https://www.mai.ru/science/trudy/published.php?ID $=35507$

4. D.S. Budaev, G.Iu. Voshchuk, N.A. Gusev et al., Coglasovannoe upravlenie gruppoi BPLA $\mathrm{s}$ primeneniem mul'tiagentny'kh tekhnologii i setecentricheskogo podhoda. E'lektronny ${ }^{`}$ ' resurs (2015) www.conf58.mipt.ru

5. V.Y. Bulychev, Y.G. Bulychev, S.S. Ivakina et al., Journal of Computer and Systems Sciences International, 54(4), 593-608 (2015) 